\title{
Shrinking of Rapidly Evaporating Water Microdroplets Reveals their Extreme Supercooling
}

\author{
Claudia Goy, ${ }_{1}^{1}$ Marco A. C. Potenza, ${ }^{2}$ Sebastian Dedera, ${ }^{3}$ Marilena Tomut, ${ }^{4}$ Emmanuel Guillerm, ${ }^{5}$ Anton Kalinin,,${ }^{1,4}$ \\ Kay-Obbe Voss, ${ }^{4}$ Alexander Schottelius, ${ }^{1}$ Nikolaos Petridis, ${ }^{4}$ Alexey Prosvetov, ${ }^{4}$ Guzmán Tejeda, ${ }^{6}$ José M. Fernández, ${ }^{6}$ \\ Christina Trautmann, ${ }^{4,7}$ Frédéric Caupin, ${ }^{5}$ Ulrich Glasmacher, ${ }^{3}$ and Robert E. Grisenti ${ }^{1,4, *}$ \\ ${ }^{1}$ Institut für Kernphysik, J. W. Goethe-Universität Frankfurt(M), 60438 Frankfurt(M), Germany \\ ${ }^{2}$ Dipartimento di Fisica, Università degli Studi di Milano, 20133 Milano, Italy \\ ${ }^{3}$ Institute of Earth Sciences, 69120 Heidelberg, Germany \\ ${ }^{4}$ GSI-Helmholtzzentrum für Schwerionenforschung, 64291 Darmstadt, Germany \\ ${ }^{5}$ Université Lyon, Université Claude Bernard Lyon 1, CNRS, Institut Lumière Matière, 69622 Lyon, France \\ ${ }^{6}$ Laboratory of Molecular Fluid Dynamics, Instituto de Estructura de la Materia, CSIC, 28006 Madrid, Spain \\ ${ }^{7}$ Material- und Geowissenschaften, Technische Universität Darmstadt, 64287 Darmstadt, Germany
}

(Received 26 May 2017; published 2 January 2018)

\begin{abstract}
The fast evaporative cooling of micrometer-sized water droplets in a vacuum offers the appealing possibility to investigate supercooled water-below the melting point but still a liquid — at temperatures far beyond the state of the art. However, it is challenging to obtain a reliable value of the droplet temperature under such extreme experimental conditions. Here, the observation of morphology-dependent resonances in the Raman scattering from a train of perfectly uniform water droplets allows us to measure the variation in droplet size resulting from evaporative mass losses with an absolute precision of better than $0.2 \%$. This finding proves crucial to an unambiguous determination of the droplet temperature. In particular, we find that a fraction of water droplets with an initial diameter of $6379 \pm 12 \mathrm{~nm}$ remain liquid down to $230.6 \pm 0.6 \mathrm{~K}$. Our results question temperature estimates reported recently for larger supercooled water droplets and provide valuable information on the hydrogen-bond network in liquid water in the hard-to-access deeply supercooled regime.
\end{abstract}

DOI: 10.1103/PhysRevLett.120.015501

Water can exist in the liquid state at temperatures far below its normal melting point. The first report on the observation of supercooled water probably dates back to Fahrenheit, who had cooled water to $264 \mathrm{~K}$ [1]. A better understanding of the properties of supercooled water [2,3] as well as establishing how and at which rates it transforms to ice [4-6] represent important goals with potentially broader impacts. For instance, tiny droplets of supercooled water at temperatures as low as $238 \mathrm{~K}$ naturally occur in the upper clouds of Earth's atmosphere [7], and an improved description of atmospheric ice formation could help to develop more reliable climate models [8]. More generally, the structural properties of supercooled water have been related to its anomalous behavior [9]. Water is an unusual liquid, because many dynamic properties (such as the viscosity and relaxation times) and thermodynamic functions (such as the heat capacity and the isothermal compressibility) show a power-law increase that becomes more pronounced in the supercooled state [10], suggesting an apparent singularity at an estimated temperature of $\approx 228 \mathrm{~K}[11,12]$. Yet what sort of singularity might water be approaching still represents an unresolved puzzle that has prompted the formulation of conflicting scenarios to interpret its origin [13].

The deeply supercooled regime is experimentally difficult to investigate because of the rapidly increasing ice nucleation rate with a decreasing temperature. Conventional techniques such as those based on the use of thin capillaries [14] or emulsions $[15,16]$ allow the study of supercooled water only at temperatures above $\approx 235 \mathrm{~K}$. In an effort to access lower temperatures, ice formation was prevented in experiments with nanoconfined water [17], aqueous solutions [18], and nanometer-scale water clusters [19]. However, the question about how the results of all these studies extrapolate to supercooled bulk water remains controversial [20].

Micrometer-sized water droplets formed in a laminar liquid jet in a vacuum offer a promising strategy to investigate supercooled bulk water and ice formation at very low temperatures [21-24]. This is due to the combination of the small sample size, suppression of heterogeneous nucleation sites, and fast evaporative cooling, with cooling rates exceeding $\sim 10^{6} \mathrm{~K} \mathrm{~s}^{-1}$ for sub-10- $\mu \mathrm{m}$-diameter water droplets. Despite its obvious benefits, this approach still lacks sufficient reliability in the determination of the droplet temperature. One conventional way of estimating the temperature of a liquid water jet in a vacuum is based on the Knudsen kinetic model of evaporative cooling [21,22,25]. This model, however, depends critically on key experimental parameters, such as the droplet diameter, which are difficult to determine with sufficient precision. Here, by Raman spectroscopy of a microscopic water jet, we obtained a 
precise measure of the evolution in the size of the rapidly evaporating droplets, thus establishing accurately their temperature and providing unambiguous evidence for the existence of supercooled bulk water down to $230.6 \mathrm{~K}$.

For a water droplet whose volume $V(t)$ and temperature $T(t)$ change with time $t$, the probability $\Sigma(T)$ to remain liquid at the temperature $T$ below the melting point $T_{m}$ is given by $[16,26]$

$$
\ln \Sigma(T) \simeq-V(0) \int_{T}^{T_{m}} \frac{J\left(T^{\prime}\right)}{\left|\dot{T}^{\prime}\right|} d T^{\prime},
$$

where $J(T)$ is the rate of ice nucleation and $|\dot{T}|$ is the cooling rate. Accordingly, the smaller the droplet size, the higher the degree of supercooling that can be reached. While nozzles having diameters larger than $10 \mu \mathrm{m}$ are routinely employed for the generation of microscopic droplet streams, nozzle clogging has severely limited the use of smaller apertures [27]. In our experiments, we injected ultrapure liquid water at $292.3 \pm 0.1 \mathrm{~K}$ into a vacuum chamber through a glass capillary nozzle with diameter $d=3.2 \pm 0.1 \mu \mathrm{m}$. We generated a periodic stream of uniform water droplets (Fig. 1) by applying an external excitation at the frequency $f=966 \mathrm{kHz}$ by means of a piezoelectric actuator to trigger the jet breakup [28].

The liquid water jet in Fig. 1 was probed by recording Raman spectra of the $\mathrm{O}-\mathrm{H}$ stretching mode as a function of the distance $z$ from the nozzle. The experimental setup was similar to that described in Ref. [29]. We employed a portable Raman instrument (iHR320, Horiba Jobin Yvon) consisting of a head for in situ analysis fiber coupled to a grating spectrometer equipped with a high-sensitivity CCD detector and providing a resolution of $\approx 1.5 \mathrm{~cm}^{-1}$. As an excitation source, we used an external Nd:YVO4 cw laser (Verdi V6, Coherent), which we operated up to a maximum power of $2.5 \mathrm{~W}$, generating a radiation beam at $\lambda_{0}=532 \mathrm{~nm}$ that was focused to $\mathrm{a} \approx 25$ - $\mu \mathrm{m}$-diameter spot onto the liquid water jet in the vacuum chamber. The Raman signal was recorded under an angle of $90^{\circ}$ with respect to the excitation laser beam and focused to the entrance of the Raman instrument's head. The Raman shifts $\Delta \nu=1 / \lambda_{0}-1 / \lambda$, where $\lambda$ is the scattered

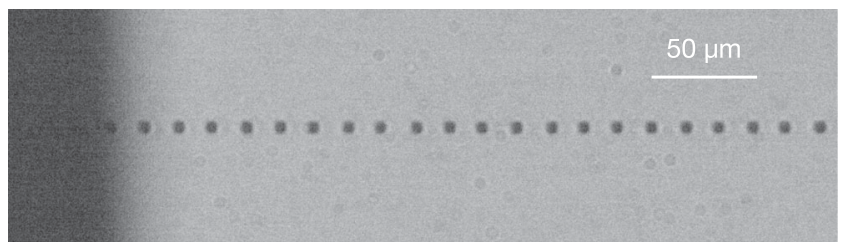

FIG. 1. Stroboscopic image of the liquid water jet emerging from a $3.2 \pm 0.1-\mu \mathrm{m}$-diameter glass capillary nozzle. The jet was illuminated by $\approx 8$-ns-long fluorescent light pulses and imaged on a $2048 \times 2048$ CCD camera equipped with a $12 \times$ objective at the working distance of $86 \mathrm{~mm}$. The jet breakup was triggered by an external excitation at the frequency of $966 \mathrm{kHz}$ to produce a periodic stream of uniform water droplets. wavelength, were calibrated by using a silicon sample. The entire liquid jet source was mounted on microactuator stages that allowed a displacement of the jet along its propagation axis with a precision of $\approx 1 \mu \mathrm{m}$. We used optical images with known dimensions to perform a zero-offset calibration of $z$ with an uncertainty of $\approx 36 \mu \mathrm{m}$.

Selected Raman spectra measured at distances between $z=0.9$ and $28.9 \mathrm{~mm}$ from the nozzle are shown in Fig. 2. During a typical acquisition time of $300 \mathrm{~s}$, up to $\sim 10^{9}$ individual droplets crossed the exciting laser beam focus. The spectrum at $28.9 \mathrm{~mm}$ in the right panel in Fig. 2 coincides with that from bulk crystalline ice [30], indicating that within our resolution all droplets froze to ice at the largest distance investigated here.

The most striking feature visible in Fig. 2 is the presence of up to five narrow peaks superimposed on the $\mathrm{O}-\mathrm{H}$ stretching bands, which progressively shift to smaller wave numbers with an increasing distance from the nozzle. These peaks originate from a morphology-dependent resonant enhancement of the Raman scattering for specific values of the droplet radius-to-wavelength ratio [31,32]. The resonances can be viewed as standing waves at the droplet-vacuum interface and require for their occurrence a perfectly smooth spherical shape. This explains the absence of the resonance peaks in the Raman spectrum from frozen droplets, which will tend to exhibit a more irregular interface with respect to the smooth surface of a liquid droplet. Accordingly, the attenuation of the resonance peaks observed at the largest distances (right panel in Fig. 2) can be interpreted as due to the rapidly decreasing fraction of purely liquid droplets.

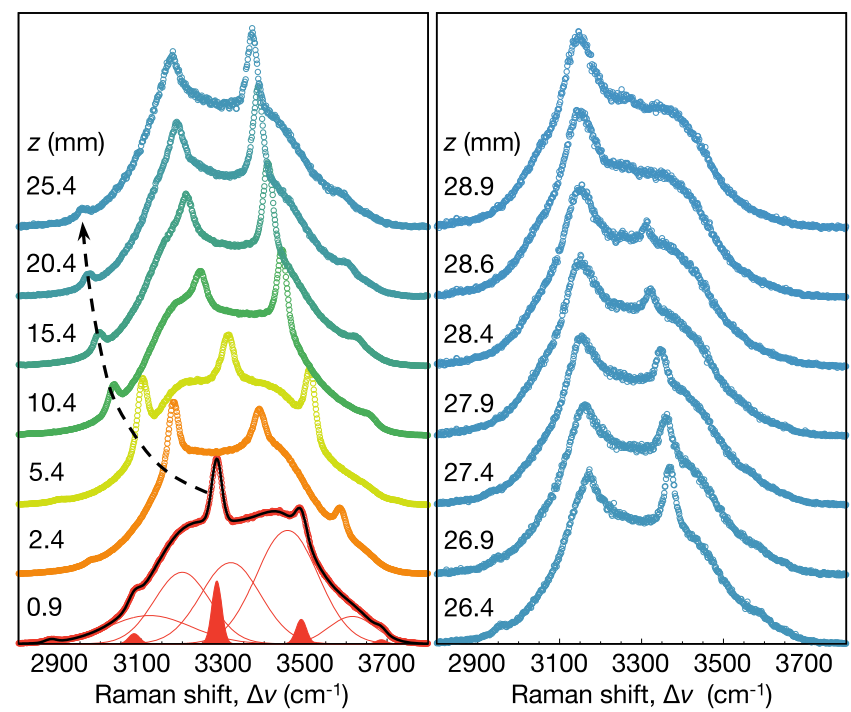

FIG. 2. Normalized and two-point baseline-corrected Raman spectra measured as a function of the distance from the nozzle, indicated by labels in both panels. The thick black solid curve is a fit to the experimental spectrum at $z=0.9 \mathrm{~mm}$ by assuming five Gaussian functions for the $\mathrm{O}-\mathrm{H}$ stretching band (shown as thin solid lines) and five additional Gaussian peaks for the resonances (shown as filled curves). 
By turning off the piezoactuator, the resonance peaks invariably disappeared, as expected for a train of droplets with a size distribution.

We now show that the observation of the resonances offers the most accurate and precise way to determine the diameter [32] and, in turn, the temperature of the evaporating water droplets. The resonances are described in the framework of the Mie-Debye light scattering theory. Relevant to the present discussion is the ratio between the extinction and the geometrical $\left(\pi r^{2}\right.$, with $r$ the droplet radius) cross sections given by [33-35]

$Q_{\text {ext }}(x, n)=\frac{2}{x^{2}} \sum_{m=1}^{\infty}(2 m+1) \operatorname{Re}\left[a_{m}(x, n)+b_{m}(x, n)\right]$,

where $x=2 \pi r / \lambda$ is the size parameter, $n$ is the refractive index, and $a_{m}(x, n)$ and $b_{m}(x, n)$ are the complex partialwave expansion scattering amplitudes. Within the size range of interest here, the function $Q_{\text {ext }}(x, n)$ exhibits a smooth ripple structure with an infinite series of sharp peaks occurring at definite values $\left\{x_{i}\right\}_{i \in \mathbb{N}}$ of the size parameter $[33,34]$. Each value corresponds to a resonance condition for a specific radius-to-wavelength ratio. Accordingly, if $x_{i}$ is the size parameter associated with any of the observed resonances centered at $\Delta \nu_{j}, j=0, \ldots, 4$, then we have

$$
\Delta \nu_{j}=\frac{1}{\lambda_{0}}-\frac{x_{i}}{2 \pi r} .
$$

Equation (3) relates the observed shift of the resonance peaks to a reduction of the droplet radius with an increasing distance from the nozzle. There is no unambiguous way to assign the size parameter $x_{i}$. However, it can be shown that the spacing $\Delta x=x_{i+2}-x_{i}$ is a very slowly varying function of the refractive index [33]. For two observed resonances centered at $\Delta \nu_{j}$ and $\Delta \nu_{j+2}$, we thus obtain from Eq. (3) for the droplet diameter

$$
D=\frac{1}{\pi} \frac{\Delta x}{\Delta \nu_{j+2}-\Delta \nu_{j}} .
$$

The Raman shifts $\Delta \nu_{j}$ were determined by fitting each Raman spectrum to five broad Gaussian components representing the fundamental $\mathrm{O}-\mathrm{H}$ stretching band $[36,37]$ and up to five additional Gaussian peaks representing the resonances; one example of such a fit is shown in Fig. 2 as a black solid curve for the spectrum measured at $z=0.9 \mathrm{~mm}$ (left panel).

The direct numerical evaluation of $Q_{\text {ext }}(x, n)$ [Eq. (2)] shows that $\Delta x=1.6396-0.6312 n$ with a standard deviation of $9.4 \times 10^{-4}$ in the range from $n=1.333$, the refractive index of liquid water at the normal melting point and $\lambda=632 \mathrm{~nm}$ [38], down to $n=1.315$ as extrapolated for supercooled water at $\approx 230 \mathrm{~K}$. For the extrapolation we adopted a modified Clausius-Mossotti relation as in the formulation by the International Association for the
Properties of Water and Steam (IAPWS), keeping the linear terms in the temperature and density $\rho$, i.e., $\left(n^{2}-1\right) /\left[\left(n^{2}+\right.\right.$ 2) $\rho]=a_{0}+a_{1} T+a_{2} \rho$ [38]. The wavelength dependence of the refractive index across the spectral Raman $\mathrm{O}-\mathrm{H}$ stretching band can be safely neglected here [38]. The coefficients $a_{0}=0.230278, a_{1}=-1.1137 \times 10^{-5}$, and $a_{2}=-0.0245171$ were determined by fitting the IAPWS expression to experimental data for the refractive index down to $258 \mathrm{~K}$ [39]. For the temperature dependence of the density of water, we assumed the sixth-order polynomial reported in Ref. [40].

In order to determine the droplet diameter as a function of the distance from the nozzle, we followed an iterative approach. Starting with an arbitrary constant value of the refractive index, we averaged Eq. (4) over the pairs of resonance peaks visible in each Raman spectrum. We performed a chi-square fit to the obtained values by using as a model function the droplet diameter $D(z)$ computed by the Knudsen theory with $D(0) \equiv D_{0}$ and the jet velocity $v$ as fit parameters. See, e.g., Ref. [22] for details of the Knudsen kinetic model. In the calculations we took into account the initial temperature gradient throughout the droplet resulting from the finite thermal conductivity of liquid water $[22,25]$. The corresponding volume-averaged droplet temperature $T(z)$ was then used to establish a new set of values of the refractive index. We repeated the above steps until convergence was reached, and the resulting values for the droplet diameter are shown as filled circles in Fig. 3(a). The thick solid line in Fig. 3(a) is the model fit with $D_{0}=6379 \pm 12 \mathrm{~nm}$ and $v=22.2 \pm 1.5 \mathrm{~m} \mathrm{~s}^{-1}$, with the $68 \%$ confidence interval indicated as a light-shaded region. The thermodynamic functions of the Knudsen model were all extrapolated from available experimental data at higher temperatures as discussed in Ref. [22]. An exception was the isobaric heat capacity, which we derived from the temperature dependence of the vapor pressure by means of Eq. (26) of Ref. [41], verifying that the result of the fit in the present temperature range was largely insensitive to our particular extrapolation [22]. We note that the above values of the fit parameters are consistent with $D_{0}=6560 \pm 370 \mathrm{~nm}$ and $v=18.3 \pm 2.0 \mathrm{~m} \mathrm{~s}^{-1}$, which we determined by using the standard relations $D^{2}=$ $3(\xi / D) d^{2} / 2$ and $v=\xi f$ [28], where $\xi$ is the (uniform) spacing between two adjacent droplets, with the ratio $\xi / D=2.88 \pm 0.27$ inferred from the stroboscopic image in Fig. 1 by simple pixel counting.

The quality of the fit in Fig. 3(a) supports the Knudsen model and the choice of the extrapolated thermodynamic parameters, providing an accurate description of the evaporative cooling process. The droplet temperature corresponding to the fit in Fig. 3(a) is shown as a thick solid curve in Fig. 3(b), with the line thickness representing the range of uncertainty. At the largest distance of $28.4 \mathrm{~mm}$ at which resonances are still visible in the Raman spectra (Fig. 2), i.e., a non-negligible fraction of droplets were still 


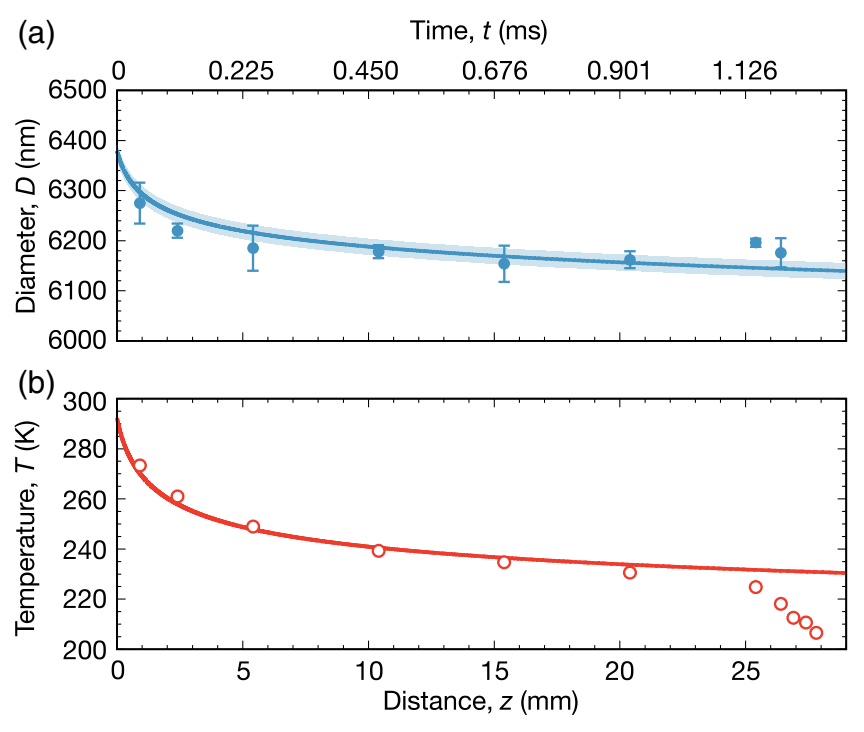

FIG. 3. Droplet diameter and temperature as a function of the distance from the nozzle, i.e., travel time $t=z / v$ (upper $x$ axis). (a) Droplet diameter determined from the Raman shifts of the resonance peaks by the iterative approach described in the main text and based on Eq. (4) (filled symbols). The error bars represent standard deviations obtained by an error propagation analysis. The thick solid curve is a fit to the data points based on the Knudsen model of evaporative cooling. The light-shaded region indicates the $68 \%$ confidence interval for the model parameters $D_{0}$ and $v$, whose standard deviations were obtained by least-squares fits to independent synthetic data sets sampled from the experimental data points [26]. (b) The thick solid curve is the calculated volumeaveraged droplet temperature corresponding to the fit shown in (a). The temperature uncertainty is represented by the line thickness. The open circles represent the droplet temperature obtained from the analysis of the shape of the Raman $\mathrm{O}-\mathrm{H}$ stretching bands shown in Fig. 4. The error bars are smaller than the symbol size.

liquid, we infer a temperature of $230.6 \pm 0.6 \mathrm{~K}$. This value represents the lowest temperature established unambiguously for supercooled bulk water.

For the purpose of comparison, we determined the droplet temperature also by the more conventional approach based on the analysis of the Raman $\mathrm{O}-\mathrm{H}$ stretching band profiles. Since the $\mathrm{O}-\mathrm{H}$ stretch vibration is a probe of the local hydrogen-bond network, it exhibits a strong variation with the temperature [42]. This dependence was exploited in the past to estimate the temperature of supercooled water droplets by Raman spectroscopy [25]. Figure 4 displays the $\mathrm{O}-\mathrm{H}$ stretching bands obtained from the Raman spectra in Fig. 2 by subtracting the resonance peak contribution. To determine the droplet temperature, we generated a calibration curve, shown in the inset in Fig. 4, by recording Raman $\mathrm{O}-\mathrm{H}$ stretching bands of liquid water enclosed in a 1-cm-wide glass cell connected to a thermostat in the temperature range 274-294 K. The inverse of the temperature plotted versus the natural logarithm of the ratio of the integrated band intensities

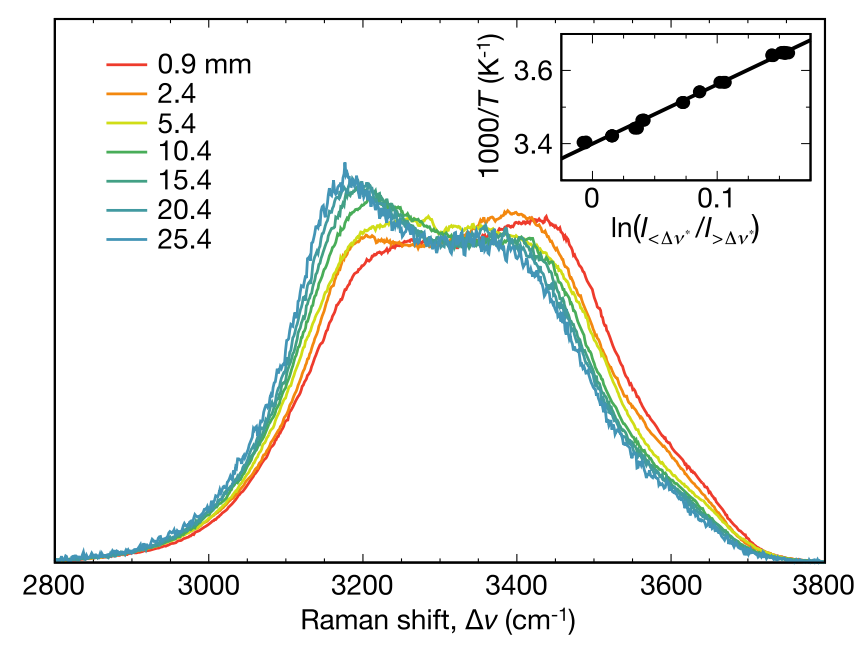

FIG. 4. Fundamental $\mathrm{O}-\mathrm{H}$ stretching bands obtained by subtracting the contribution of the resonances from the Raman spectra in Fig. 2. For clarity, only the bands up to $z=25.4 \mathrm{~mm}$ are shown. The inset shows the calibration data (filled circles) plotted as the inverse of the temperature against the natural logarithm of the ratio of the integrated band intensities $I_{<\Delta \nu^{*}}$ and $I_{>\Delta \nu^{*}}$ below and above $\Delta \nu^{*}=3360 \mathrm{~cm}^{-1}$, respectively. The solid line is a linear fit that was extrapolated to the supercooled region in order to determine the droplet temperature from the bands in the main figure.

below and above an arbitrary point close to the center of the $\mathrm{O}-\mathrm{H}$ stretching band yields a linear relationship that we extrapolated to the supercooled liquid state [25]. Additional Raman measurements performed on a liquid water sample contained in a $\approx 20-\mu$ m-inner-diameter glass capillary tube by using a second Raman instrument at the Institut Lumière Matière confirmed the validity of the established linear relationship down to $238.4 \mathrm{~K}$.

The droplet temperature estimated from the variation of the shape of the Raman $\mathrm{O}-\mathrm{H}$ stretching bands is shown in Fig. 3(b) as open circles. We find a good agreement with the temperature curve obtained from the analysis of the resonance peaks up to $z=20.4 \mathrm{~mm}$. The deviations observed for $z \gtrsim 25 \mathrm{~mm}$ are likely due to the growing contribution of the scattering from droplets that froze to ice at such large distances, affecting the shape of the $\mathrm{O}-\mathrm{H}$ stretching band. We note that the agreement in Fig. 3(b) implicitly extends the range of validity of the temperature calibration curve (inset in Fig. 4) down to $\approx 234$ K. Overall, the established consistency between the two distinct approaches provides a rigorous proof of the reliability of our droplet temperature determination.

The lowest temperature reported here for $\approx 6-\mu$ m-diameter droplets [Fig. 3(b)] is not consistent with recent temperature estimates based on the Knudsen model for nominal $\approx 12-\mu \mathrm{m}$ diameter water droplets probed with ultrashort X-ray laser pulses [22]. Indicating with $\Sigma_{12 \mu \mathrm{m}}$ and $\Sigma_{6 \mu \mathrm{m}}$ the probabilities to observe 12- and $6-\mu \mathrm{m}$-diameter water droplets, respectively, in the supercooled state at $230.6 \mathrm{~K}$, and by taking into 
account that the evaporative cooling rate increases with a decreasing droplet diameter, from Eq. (1) it follows that $\Sigma_{12 \mu \mathrm{m}} \lesssim\left(\Sigma_{6 \mu \mathrm{m}}\right)^{8} \ll \Sigma_{6 \mu \mathrm{m}} \ll 1$. However, in Ref. [22], it was estimated that nearly $100 \%$ of the $12-\mu$ m-diameter droplets were liquid at $230.6 \mathrm{~K}$, and a fraction of them were even reported to have supercooled further to $227 \mathrm{~K}$. We note that in our experiments the heating of the droplets by the excitation laser beam was completely negligible because of the extremely short transit time across the laser beam focus of $\approx 10^{-6} \mathrm{~s}$ and the small absorption cross section at $532 \mathrm{~nm}$ of $0.0447 \mathrm{~m}^{-1}$. Although ice nucleation can be triggered by short, 532-nm laser pulses at the intensity threshold of $\sim 10^{16} \mathrm{~W} \mathrm{~m}^{-2}$ [43], this phenomenon unlikely occurred in the present study because of the several orders of magnitude lower cw-laser intensity of $\approx 5 \times 10^{9} \mathrm{~W} \mathrm{~m}^{-2}$. Thus, while the above apparent discrepancy can be conclusively resolved only by a direct comparison between the two (Raman and $\mathrm{x}$-ray) scattering techniques, our results indicate that the degree of supercooling of micrometer-sized water droplets investigated recently might be largely overestimated, thereby challenging the interpretation of the reported experimental data $[22-24,44,45]$.

Vibrational spectroscopy has been widely applied to the study of the structure of bulk liquid water [46], evidencing, in particular, a continuous evolution in the Raman spectral features from ambient to supercooled conditions $[36,37,47]$. With a decreasing temperature, the low-frequency side of the $\mathrm{O}-\mathrm{H}$ stretching band around $3200 \mathrm{~cm}^{-1}$ becomes more pronounced with respect to the high-frequency side around $3400 \mathrm{~cm}^{-1}$. One interpretation attributes this behavior to the change upon cooling in the population of two distinct local hydrogen-bond structures-distorted and tetrahedral-associated with the high- and low-frequency spectral branches, respectively [9]. The Raman $\mathrm{O}-\mathrm{H}$ stretching bands shown in Fig. 4 clearly confirm this trend, indicating that it further extends down to at least $\approx 232 \mathrm{~K}$. No definite conclusion can be drawn here on whether this trend continues at even lower temperatures because of the scattering from frozen droplets for $z \gtrsim 25 \mathrm{~mm}$. More insights in this respect may come from the low-energy vibrations involving intermolecular hydrogen bonds. Recent time-resolved optical Kerr effect measurements identified clear signatures of two structural components in the low-frequency spectral region around $200 \mathrm{~cm}^{-1}$ [48]. However, these experiments were carried out at temperatures above $247 \mathrm{~K}$, which is much higher than the lowest temperatures reported here. By probing the water droplets of the present study in the low-frequency region, it would thus be possible to elucidate the nature of the structural evolution and ice formation occurring in liquid water in the deeply supercooled regime.

This work was in part supported by Bundesministerium für Bildung und Forschung through Grant No. 05K13RF5. grisenti@atom.uni-frankfurt.de

[1] D. G. Fahrenheit, Phil. Trans. R. Soc. London 33, 78 (1724).

[2] P. H. Poole, F. G. Sciortino, U. Essmann, and H. E. Stanley, Nature (London) 360, 324 (1992).

[3] J. C. Palmer, F. Martelli, Y. Liu, R. Car, A. Z. Panagiotopoulos, and P. G. Debenedetti, Nature (London) 510, 385 (2014).

[4] E. Moore and V. Molinero, Nature (London) 479, 506 (2011).

[5] J. Russo, F. Romano, and H. Tanaka, Nat. Mater. 13, 733 (2014).

[6] A. Haji-Akbari and P. G. Debenedetti, Proc. Natl. Acad. Sci. U.S.A. 112, 10582 (2015).

[7] D. Rosenfeld and W. L. Woodley, Nature (London) 405,440 (2000).

[8] B. J. Murray, D. O'Sullivan, J. D. Atkinson, and M.E. Webb, Chem. Soc. Rev. 41, 6519 (2012).

[9] A. Nilsson and G. M. Pettersson, Nat. Commun. 6, 8998 (2015).

[10] P. G. Debenedetti, J. Phys. Condens. Matter 15, R1669 (2003).

[11] R. J. Speedy and C. A. Angell, J. Chem. Phys. 65, 851 (1976).

[12] A. Dehaoui, B. Issenmann, and F. Caupin, Proc. Natl. Acad. Sci. U.S.A. 112, 12020 (2015).

[13] P. Gallo, K. Amann-Winkel, C. A. Angell, M. A. Anisimov, F. Caupin, C. Chakravarty, E. Lascaris, T. Loerting, A. Z. Panagiotopoulos, J. Russo, J. A. Sellberg, H. E. Stanley, H. Tanaka, C. Vega, L. Xu, and L. G. M. Pettersson, Chem. Rev. 116, 7463 (2016).

[14] D. E. Hare and C. M. Sorensen, J. Chem. Phys. 84, 5085 (1986).

[15] P. Taborek, Phys. Rev. B 32, 5902 (1985).

[16] B. Riechers, F. Wittbracht, A. Hütten, and T. Koop, Phys. Chem. Chem. Phys. 15, 5873 (2013).

[17] F. Mallamace, C. Corsaro, and H. E. Stanley, Proc. Natl. Acad. Sci. U.S.A. 110, 4899 (2013).

[18] K.-I. Murata and H. Tanaka, Nat. Mater. 11, 436 (2012).

[19] A. Manka, H. Pathak, S. Tanimura, J. Wölk, R. Strey, and B. E. Wyslouzil, Phys. Chem. Chem. Phys. 14, 4505 (2012).

[20] F. Caupin, J. Non-Cryst. Solids 407, 441 (2015).

[21] M. Faubel, S. Schlemmer, and J. P. Toennies, Z. Phys. D 10, 269 (1988).

[22] J. A. Sellberg, C. Huang, T. A. McQueen, N. D. Loh, H. Laksmono, D. Schlesinger, R. G. Sierra, D. Nordlund, C. Y. Hampton, D. Starodub, D. P. DePonte, M. Beye, C. Chen, A. V. Martin, A. Barty, K. T. Wikfeldt, T. M. Weiss, C. Caronna, J. Feldkamp, L. B. Skinner, M. M. Seibert, M. Messerschmidt, G. J. Williams, S. Boutet, L. G. M. Pettersson, M. J. Bogan, and A. Nilsson, Nature (London) 510, 381 (2014).

[23] J. A. Sellberg, T. A. McQueen, H. Laksmono, S. Schreck, M. Beye, D. P. DePonte, B. Kennedy, D. Nordlund, R. G. Sierra, D. Schlesinger, T. Tokushima, I. Zhovtobriukh, S. Eckert, V. H. Segtnan, H. Ogasawara, K. Kubicek, S. Techert, U. Bergmann, G. L. Dakovski, W. F. Schlotter, Y. Harada, M. J. Bogan, P. Wernet, A. Föhlisch, L. G. M. Pettersson, and A. Nilsson, J. Chem. Phys. 142, 044505 (2015).

[24] H. Laksmono, T. A. McQueen, J. A. Sellberg, N. D. Loh, C. Huang, D. Schlesinger, R. G. Sierra, C. Y. Hampton, D. Nordlund, M. Beye, A. V. Martin, A. Barty, M. M. Seibert, M. Messerschmidt, G. J. Williams, S. Boutet, K. Amann-Winkel, T. Loerting, L. G. M. Pettersson, M. J. Bogan, and A. Nilsson, J. Phys. Chem. Lett. 6, 2826 (2015). 
[25] J. D. Smith, C. D. Cappa, W. S. Drisdell, R. C. Cohen, and R. J. Saykally, J. Am. Chem. Soc. 128, 12892 (2006).

[26] M. E. Mekki-Azouzi, C. Ramboz, J.-F. Lenain, and F. Caupin, Nat. Phys. 9, 38 (2013).

[27] D. P. DePonte, U. Weierstall, K. Schmidt, J. Warner, D. Starodub, J. C. H. Spence, and R. B. Doak, J. Phys. D 41, 195505 (2008).

[28] A. Frohn and N. Roth, Dynamics of Droplets (Springer, Berlin, 2000).

[29] M. Kühnel, J. M. Fernández, G. Tejeda, A. Kalinin, S. Montero, and R. E. Grisenti, Phys. Rev. Lett. 106, 245301 (2011).

[30] C. Q. Sun, X. Zhang, X. Fu, W. Zheng, J.-1. Kuo, Y. Zhou, Z. Shen, and J. Zhou, J. Phys. Chem. Lett. 4, 3238 (2013).

[31] R. Thurn and W. Kiefer, Appl. Opt. 24, 1515 (1985).

[32] W. Kiefer, J. Popp, M. Lankers, M. Trunk, I. Hartmann, E. Urlaub, and J. Musick, J. Mol. Struct. 408, 113 (1996).

[33] P. Chýlek, J. Opt. Soc. Am. 66, 285 (1976).

[34] P. Chýlek, J. T. Kiehl, and M. K. W. Ko, Phys. Rev. A 18, 2229 (1978).

[35] C. F. Bohren and D. R. Huffman, Absorption and Scattering of Light by Small Particles (Wiley, Berlin, 1998).
[36] H. Suzuki, Y. Matsuzaki, A. Muraoka, and M. Tachikawa, J. Chem. Phys. 136, 234508 (2012).

[37] Q. Sun, Chem. Phys. Lett. 568-569, 90 (2013).

[38] A. H. Harvey, J. S. Gallagher, and J. M. H. Levelt Sengers, J. Phys. Chem. Ref. Data 27, 761 (1998).

[39] L. Carroll and M. Henry, Appl. Opt. 41, 1330 (2002).

[40] D. E. Hare and C. M. Sorensen, J. Chem. Phys. 87, 4840 (1987).

[41] J. Kalova and R. Mares, Int. J. Thermophys. 31, 756 (2010).

[42] G. E. Walrafen, J. Chem. Phys. 47, 114 (1967).

[43] F. Aliotta, P. V. Giaquinta, R. C. Ponterio, S. Prestipino, F. Saija, G. Salvato, and C. Vasi, Sci. Rep. 4, 7230 (2014).

[44] T. Koop and B. J. Murray, J. Chem. Phys. 145, 211915 (2016).

[45] H. Pathak, J. C. Palmer, D. Schlesinger, K. T. Wikfeldt, J. A. Sellberg, L. G. M. Pettersson, and A. Nilsson, J. Chem. Phys. 145, 134507 (2016).

[46] H. J. Bakker and J. L. Skinner, Chem. Rev. 110, 1498 (2010).

[47] D. E. Hare and C. M. Sorensen, J. Chem. Phys. 93, 25 (1990).

[48] A. Taschin, P. Bartolini, R. Eramo, R. Righini, and R. Torre, Nat. Commun. 4, 2401 (2013). 